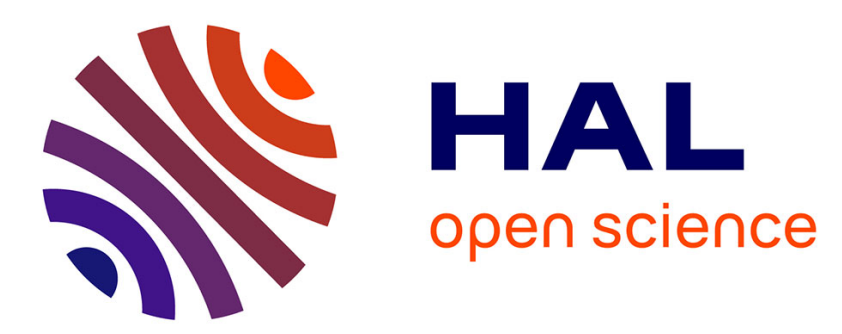

\title{
A Tractable Closed-Form Expression of the Coverage Probability in Poisson Cellular Networks
}

Marco Di Renzo, Thanh Tu Lam, Alessio Zappone, Mérouane Debbah

\section{To cite this version:}

Marco Di Renzo, Thanh Tu Lam, Alessio Zappone, Mérouane Debbah. A Tractable Closed-Form Expression of the Coverage Probability in Poisson Cellular Networks. IEEE Wireless Communications Letters, 2018, 8 (1), pp.249-252. 10.1109/LWC.2018.2868753 . hal-01880303

\section{HAL Id: hal-01880303 https://hal.science/hal-01880303}

Submitted on 7 Jul 2020

HAL is a multi-disciplinary open access archive for the deposit and dissemination of scientific research documents, whether they are published or not. The documents may come from teaching and research institutions in France or abroad, or from public or private research centers.
L'archive ouverte pluridisciplinaire HAL, est destinée au dépôt et à la diffusion de documents scientifiques de niveau recherche, publiés ou non, émanant des établissements d'enseignement et de recherche français ou étrangers, des laboratoires publics ou privés. 


\title{
A Tractable Closed-Form Expression of the Coverage Probability in Poisson Cellular Networks
}

\author{
Marco Di Renzo, Senior Member, IEEE, Thanh Tu Lam, Student Member, IEEE \\ Alessio Zappone, Senior Member, IEEE, and Mérouane Debbah, Fellow, IEEE
}

\begin{abstract}
The coverage of cellular networks is usually defined as the probability that the Signal-to-Interference+Noise-Ratio (SINR) is greater than a reliability threshold. Based on this definition, the coverage cannot, in general, be formulated in a tractable closed-form expression. In [1], the authors have introduced a new definition of coverage that is shown to be mathematically tractable for system-level analysis and optimization. In this letter, we conduct a thorough comparison between the two definitions of coverage and show that the coverage introduced in [1] provides one with a tractable and closed-form approximation for the SINR-coverage, which is proved to be an upper-bound in relevant operating regimes. By using the new definition of coverage, the impact of several network parameters, e.g., the density of base stations and mobile terminals, the transmit power, and the transmission bandwidth, can be analytically proved.
\end{abstract}

Index Terms-Cellular networks, stochastic geometry.

\section{INTRODUCTION}

During the last few years, stochastic geometry tools, and, in particular, the theory of Poisson Point Processes (PPPs) have been widely used for modeling and analyzing cellular networks [2]. By assuming Base Stations (BSs) and Mobile Terminals (MTs) distributed according to two independent homogeneous PPPs, a cell association criterion based on the highest average received power, a singular path-loss model, Rayleigh fading, as well as BSs and MTs equipped with a single antenna, the authors of [3] have obtained the following tractable expression of the coverage probability $\left(\mathrm{P}_{\text {cov }}\right)$ :

$$
\mathrm{P}_{\mathrm{cov}}^{(\mathrm{SINR})}\left(\gamma_{\mathrm{D}}\right)=\operatorname{Pr}\left\{\mathrm{SINR} \geq \gamma_{\mathrm{D}}\right\}=\pi \lambda_{\mathrm{BS}} \int_{0}^{+\infty} e^{-f(x)} d x
$$

where symbols and notation in Table I are used, SINR = $\mathcal{U} /\left(\sigma_{\mathrm{N}}^{2}+\mathcal{I}\right)$ is the Signal-to-Interference+Noise-Ratio, and $f(x)=\left(\kappa \sigma_{\mathrm{N}}^{2} / \mathrm{P}_{\mathrm{tx}}\right) \gamma_{\mathrm{D}} x^{\beta / 2}+\pi \lambda_{\mathrm{BS}}\left(1+\Upsilon \mathcal{L}\left(\lambda_{\mathrm{MT}} / \lambda_{\mathrm{BS}}\right)\right) x$.

The SINR-coverage in (1) cannot, in general, be formulated in closed-form. An exception is the interference-limited regime, where $\mathrm{P}_{\mathrm{cov}}^{(\mathrm{SINR})}\left(\gamma_{\mathrm{D}}\right)=\mathrm{P}_{\mathrm{cov}}^{(\mathrm{SIR})}\left(\gamma_{\mathrm{D}}\right)=\operatorname{Pr}\left\{\mathrm{SIR} \geq \gamma_{\mathrm{D}}\right\}$ $=\left(1+\Upsilon \mathcal{L}\left(\lambda_{\mathrm{MT}} / \lambda_{\mathrm{BS}}\right)\right)^{-1}$, and $\mathrm{SIR}=\mathcal{U} / \mathcal{I}$ is the Signal-toInterference-Ratio. The SIR-coverage is independent of $\mathrm{P}_{\mathrm{tx}}$, which may lead to meaningless optimization problems [1].

To overcome these limitations, the authors of [1] have introduced a new definition of coverage, which accounts for the sensitivity of the MTs during both the data detection and

Manuscript received April 4, 2018. This work was supported in part by the European Commission through the H2020-MSCA ETN-5Gwireless project under grant 641985, the H2020-MSCA IF-BESMART project under grant 749336, and the H2020-ERC PoC-CacheMire project under grant 727682.

The authors are with the Laboratoire des Signaux et Systèmes, CNRS, CentraleSupélec, Univ Paris Sud, Université Paris-Saclay, 3 rue Joliot Curie, Plateau du Moulon, 91192 Gif-sur-Yvette, France. (e-mail: marco.direnzo@12s.centralesupelec.fr).
TABLE I

SYMBOLS AND NOTATION $\left(\alpha=3.5, \delta=2 / \beta, \beta>2, \eta=\kappa \sigma_{\mathrm{N}}^{2} \gamma_{\mathrm{A}}\right)$. ALso: $\overline{\mathcal{U}}=\mathrm{P}_{\mathrm{tx}} / \ell\left(r_{0}\right), \mathcal{U}=g_{0} \mathrm{P}_{\mathrm{tx}} / \ell\left(r_{0}\right)$, $\mathcal{I}=\sum_{r_{i} \in \Psi_{\mathrm{BS}} \backslash r_{0}} g_{i} \mathrm{P}_{\mathrm{tx}} / \ell\left(r_{i}\right), \operatorname{Pr}\left\{r_{0} \leq r\right\}=1-e^{-\pi \lambda_{\mathrm{BS}} r^{2}}$, $\operatorname{Pr}\left\{g_{0} \leq g\right\}=\operatorname{Pr}\left\{g_{i} \leq g\right\}=1-e^{-g}$, $\Upsilon={ }_{2} F_{1}\left(-\delta, 1,1-\delta,-\gamma_{\mathrm{D}}\right)-1 \geq 0, \mathcal{L}(x)=1-(1+x / \alpha)^{-\alpha}$, $\mathcal{Q}(x, y, z)=1-\exp \left(-\pi x(y / \eta)^{\delta}(1+\Upsilon \mathcal{L}(z))\right)$.

\begin{tabular}{|l||l|}
\hline \multicolumn{1}{|c|}{ Symbol/Function } & \multicolumn{1}{c|}{ Definition } \\
\hline \hline $\mathbb{E}\{\cdot\}, \operatorname{Pr}\{\cdot\}$ & Expectation, probability measure \\
\hline$\Psi_{\mathrm{BS}}, \lambda_{\mathrm{BS}}, \lambda_{\mathrm{MT}}$ & PPP and density of BSs, density of MTs \\
\hline $\mathrm{P}_{\mathrm{tx}}, \mathrm{B}_{\mathrm{W}}$ & Transmit power, transmission bandwidth \\
\hline $\mathrm{N}_{0}, \sigma_{\mathrm{N}}^{2}=\mathrm{B}_{\mathrm{W}} \mathrm{N}_{0}$ & Noise power density, noise variance \\
\hline$r_{0}, g_{0}$ & Distance and fading power of serving BS \\
\hline$r_{i}, g_{i}$ & Distance and fading power of interfering BSs \\
\hline$\ell(r)=\kappa r^{\beta}, \kappa, \beta$ & Path-loss function, constant, slope \\
\hline$\gamma_{\mathrm{D}}, \gamma_{\mathrm{A}}$ & Threshold for decoding, cell association \\
\hline${ }_{2} F_{1}(\cdot, \cdot, \cdot, \cdot)$ & Gauss hypergeometric function \\
\hline$\Gamma(\cdot)$, erfc $(\cdot)$ & Gamma function, complementary error function \\
\hline $\mathrm{P}_{\mathrm{cov}}, \mathrm{PSE}$ & Coverage probability, potential spectral efficiency \\
\hline$\dot{z}_{x}(x, \cdot), \ddot{z}_{x}(x, \cdot)$ & 1st and 2nd derivative with respect to $x$ \\
\hline
\end{tabular}

cell association phases. Based on [1], the coverage probability can be formulated in a tractable closed-form expression:

$$
\begin{aligned}
& \mathrm{P}_{\mathrm{cov}}\left(\gamma_{\mathrm{D}}, \gamma_{\mathrm{A}}\right)=\operatorname{Pr}\left\{\mathrm{SIR} \geq \gamma_{\mathrm{D}}, \mathrm{ASNR} \geq \gamma_{\mathrm{A}}\right\} \\
&= \pi \lambda_{\mathrm{BS}} \int_{0}^{\left(\mathrm{P}_{\mathrm{tx}} / \eta\right)^{\delta}} e^{-\pi \lambda_{\mathrm{BS}}\left(1+\Upsilon \mathcal{L}\left(\lambda_{\mathrm{MT}} / \lambda_{\mathrm{BS}}\right)\right) x} d x \\
& \quad=\mathcal{Q}\left(\lambda_{\mathrm{BS}}, \mathrm{P}_{\mathrm{tx}}, \lambda_{\mathrm{MT}} / \lambda_{\mathrm{BS}}\right)\left(1+\Upsilon \mathcal{L}\left(\lambda_{\mathrm{MT}} / \lambda_{\mathrm{BS}}\right)\right)^{-1}
\end{aligned}
$$

where ASNR $=\overline{\mathcal{U}} / \sigma_{\mathrm{N}}^{2}$ is the Signal-to-Noise-Ratio (SNR) averaged over Rayleigh fading, which accounts for the smallest average SNR needed to ensure a successful cell association.

Problem Statement: Unlike the SINR-coverage that cannot be formulated in closed-form and the SIR-coverage that is independent of key system parameters, the coverage in (2) is given in closed-form, depends on the most relevant network parameters, and is proved in [1] to be simple enough, tractable, and meaningful for system optimization. Motivated by these strengths, this letter has four objectives: i) to identify a suitable choice of $\gamma_{\mathrm{A}}$ that makes (2) a good approximation of (1), ii) to evaluate the accuracy of the proposed approximation, iii) to study the performance trends of (2) as a function of key parameters to optimize cellular networks, i.e., $\mathrm{P}_{\mathrm{tx}}, \mathrm{B}_{\mathrm{W}}, \lambda_{\mathrm{BS}}$, $\lambda_{\mathrm{MT}}$, and iv) to evaluate whether the proposed approximation results in the same performance trends as the SINR-coverage.

\section{Proposed Approximation}

The rationale of the proposed approximation consists of ensuring that (1) and (2) coincide with (or are asymptotically close to) each other when the corresponding network models operate in their own interference-limited and noise-limited regimes. As for (1), these regimes correspond to $\mathrm{P}_{\mathrm{cov}}^{(\mathrm{SINR})}\left(\gamma_{\mathrm{D}}\right)=\operatorname{Pr}\left\{\mathrm{SIR} \geq \gamma_{\mathrm{D}}\right\}$ and $\mathrm{P}_{\mathrm{cov}}^{\text {(SINR) }}\left(\gamma_{\mathrm{D}}\right)=$ 
$\operatorname{Pr}\left\{\mathrm{SNR} \geq \gamma_{\mathrm{D}}\right\}$, respectively. As for (2), they correspond to $\mathrm{P}_{\text {cov }}\left(\gamma_{\mathrm{D}}, \gamma_{\mathrm{A}}\right)=\operatorname{Pr}\left\{\mathrm{SIR} \geq \gamma_{\mathrm{D}}\right\}$ and $\mathrm{P}_{\mathrm{cov}}\left(\gamma_{\mathrm{D}}, \gamma_{\mathrm{A}}\right)=$ $\operatorname{Pr}\left\{\mathrm{ASNR} \geq \gamma_{\mathrm{A}}\right\}$, respectively. Thus, the following holds.

In the interference-limited regime, i.e., $\sigma_{\mathrm{N}}^{2}=0$, we obtain, by direct inspection of (1) and (2), $\mathrm{P}_{\mathrm{Cov}}^{(\mathrm{SINR})}\left(\gamma_{\mathrm{D}}\right)=$ $\mathrm{P}_{\text {cov }}\left(\gamma_{\mathrm{D}}, \gamma_{\mathrm{A}}\right)=\mathrm{P}_{\mathrm{cov}}^{(\mathrm{SIR})}\left(\gamma_{\mathrm{D}}\right)$, which holds true for any $\gamma_{\mathrm{A}}$.

In the noise-limited regime, i.e., $\mathcal{I}=0$, (1) and (2) are:

$$
\begin{aligned}
& \mathrm{P}_{\text {cov }}^{(\mathrm{SINR})}\left(\gamma_{\mathrm{D}}\right)= \operatorname{Pr}\left\{\mathrm{SNR} \geq \gamma_{\mathrm{D}}\right\} \\
& \stackrel{(a)}{=} \mathbb{E}_{g_{0}}\left\{\operatorname{Pr}\left\{r_{0} \leq\left(g_{0} r_{\mathrm{D}}\right)^{1 / \beta}\right\}\right\} \stackrel{(b)}{\leq} 1-e^{-\pi \lambda_{\mathrm{BS}} \Gamma(1+\delta) r_{\mathrm{D}}^{\delta}} \\
& \mathrm{P}_{\mathrm{cov}}\left(\gamma_{\mathrm{D}}, \gamma_{\mathrm{A}}\right)=\operatorname{Pr}\left\{\mathrm{ASNR} \geq \gamma_{\mathrm{A}}\right\} \\
& \stackrel{(a)}{=} \operatorname{Pr}\left\{r_{0} \leq r_{\mathrm{A}}^{1 / \beta}\right\}=1-e^{-\pi \lambda_{\mathrm{BS}} r_{\mathrm{A}}^{\delta}}
\end{aligned}
$$

where $r_{\mathrm{D}}=\mathrm{P}_{\mathrm{tx}} /\left(\kappa \sigma_{\mathrm{N}}^{2} \gamma_{\mathrm{D}}\right), r_{\mathrm{A}}=\mathrm{P}_{\mathrm{tx}} /\left(\kappa \sigma_{\mathrm{N}}^{2} \gamma_{\mathrm{A}}\right)$, (a) follows from the definitions of SNR and ASNR, and (b) follows by applying the Jensen's inequality and $\mathbb{E}_{g_{0}}\left\{g_{0}^{\delta}\right\}=\Gamma(1+\delta)$.

Remark 1: Jensen's inequality is necessary in order to have a tractable closed-form expression of (3) and to avoid using special functions, e.g., the Meijer's G-function. By direct inspection, it is easy to show that the tightness of the upperbound in (3) increases as either $\lambda_{\mathrm{BS}}$ or $\mathrm{P}_{\mathrm{tx}}$ decrease. Thus, (3) is expected to be accurate in the noise-limited regime.

Approach: The SINR-coverage in (1) is approximated as:

$$
\mathrm{P}_{\operatorname{cov}}^{(\mathrm{SINR})}\left(\gamma_{\mathrm{D}}\right) \approx \mathrm{P}_{\mathrm{cov}}\left(\gamma_{\mathrm{D}}, \gamma_{\mathrm{A}}=\gamma_{\mathrm{D}} \Gamma(1+\delta)^{-1 / \delta}\right)
$$

Rationale: Equation (5) follows by equating (3) and (4), which ensures that (2) is a tight upper-bound of (1) if $\mathcal{I}=0$.

Remark 2: Based on (5), $\gamma_{\mathrm{A}}=\gamma_{\mathrm{D}} \Gamma(1+\delta)^{-1 / \delta}$. Arguably, this is a suitable result for system-level optimization, since $\gamma_{\mathrm{A}}$ depends only on $\beta$ and $\gamma_{\mathrm{D}}$, while it is constant as a function of the key parameters usually used to optimize cellular networks, i.e., $\mathrm{P}_{\mathrm{tx}}, \mathrm{B}_{\mathrm{W}}, \lambda_{\mathrm{BS}}, \lambda_{\mathrm{MT}}$. Based on (5), e.g., the conclusions drawn in [1] about the optimal $\mathrm{P}_{\mathrm{tx}}$ and $\lambda_{\mathrm{BS}}$ that maximize the energy efficiency based on (2) apply unaltered to (1).

\section{A. Expected Accuracy}

From the previous section, we evince that the approximation in (5) is exact in the interference-limited regime and is a tight upper-bound in the noise-limited regime. In this section, we study the accuracy of (5) beyond these asymptotic regimes. Due to the lack of a closed-form expression for (1), this is a challenging task for arbitrary system setups. Then, we consider two case studies for which the integral in (1) can be computed.

Lemma 1: If $\beta=4$, the proposed approximation in (5) is an upper-bound of the SINR-coverage in (1), i.e., $\mathrm{P}_{\text {cov }}^{(\mathrm{SINR})}\left(\gamma_{\mathrm{D}}\right) \leq$ $\mathrm{P}_{\text {cov }}\left(\gamma_{\mathrm{D}}, \gamma_{\mathrm{A}}=\gamma_{\mathrm{D}} \Gamma(1+\delta)^{-1 / \delta}\right)$.

Proof: Let $\mathcal{A}=(1 / 2) \pi \lambda_{\mathrm{BS}}\left(1+\Upsilon \mathcal{L}\left(\lambda_{\mathrm{MT}} / \lambda_{\mathrm{BS}}\right)\right) r_{\mathrm{D}}^{1 / 2} \geq$ 0 , the SINR-coverage in (1) simplifies to $\mathrm{P}_{\mathrm{Cov}}^{(\mathrm{SINR})}\left(\gamma_{\mathrm{D}}\right)=$ $\sqrt{\pi}\left(1+\Upsilon \mathcal{L}\left(\lambda_{\mathrm{MT}} / \lambda_{\mathrm{BS}}\right)\right)^{-1} \mathcal{A e r f c}(\mathcal{A}) e^{\mathcal{A}^{2}}$ and (2) reduces to $\mathrm{P}_{\mathrm{cov}}\left(\gamma_{\mathrm{D}}, \gamma_{\mathrm{A}}=\gamma_{\mathrm{D}} \Gamma(1+\delta)^{-1 / \delta}\right)=\left(1-e^{-2 \Gamma(3 / 2) \mathcal{A}}\right)$ $\times\left(1+\Upsilon \mathcal{L}\left(\lambda_{\mathrm{MT}} / \lambda_{\mathrm{BS}}\right)\right)^{-1}$. The proof follows, since $\sqrt{\pi} \mathcal{A} \operatorname{erfc}(\mathcal{A}) e^{\mathcal{A}^{2}}+e^{-2 \Gamma(3 / 2) \mathcal{A}} \leq 1$ for $\mathcal{A} \geq 0$.

Lemma 2: In the low-noise regime, i.e., $\sigma_{\mathrm{N}}^{2} \ll \mathcal{I}$ but $\sigma_{\mathrm{N}}^{2} \neq$ 0 , the approximation in (5) is an upper-bound of (1).
Proof: In the low-noise regime, the approximation $e^{-\left(1 / r_{\mathrm{D}}\right) x^{1 / \delta}} \approx 1-\left(1 / r_{\mathrm{D}}\right) x^{1 / \delta}$ can be used and (1) becomes:

$$
\begin{aligned}
\mathrm{P}_{\mathrm{cov}}^{(\mathrm{SINR})}\left(\gamma_{\mathrm{D}}\right) & \approx \mathrm{P}_{\mathrm{cov}}\left(\gamma_{\mathrm{D}}, \gamma_{\mathrm{A}}=\gamma_{\mathrm{D}} \Gamma(1+\delta)^{-1 / \delta}\right) \\
& +\frac{e^{-\mathcal{B}}-\Gamma(1+1 / \delta) \Gamma(1+\delta)^{1 / \delta} \mathcal{B}^{-1 / \delta}}{1+\Upsilon \mathcal{L}\left(\lambda_{\mathrm{MT}} / \lambda_{\mathrm{BS}}\right)}
\end{aligned}
$$

with $\mathcal{B}=\pi \lambda_{\mathrm{BS}}\left(1+\Upsilon \mathcal{L}\left(\lambda_{\mathrm{MT}} / \lambda_{\mathrm{BS}}\right)\right) r_{\mathrm{A}}^{\delta} \geq 0$. Consider the function $\mathcal{B}^{1 / \delta} e^{-\mathcal{B}}$. It is positive and attains its maximum at $\mathcal{B}=1 / \delta$. Thus, $e^{-\mathcal{B}} \mathcal{B}^{1 / \delta} \Gamma(1+\delta)^{-1 / \delta} \Gamma(1+1 / \delta)^{-1} \leq$ $(1 / \delta)^{1 / \delta} e^{-1 / \delta} \Gamma(1+\delta)^{-1 / \delta} \Gamma(1+1 / \delta)^{-1} \leq 1$ for $\beta>2$. So, the second line of (6) is negative and the proof follows.

\section{B. Interplay of Transmit Power and Density of Base Stations}

Given a desired coverage probability, $\mathrm{P}_{\mathrm{cov}}^{*}$, to be fulfilled, three questions are of importance for the design and optimization of cellular networks: i) Is $\mathrm{P}_{\text {cov }}^{*}$ achievable? ii) If so, what is the functional relation between $\mathrm{P}_{\mathrm{tx}}$ and $\lambda_{\mathrm{BS}}$ to achieve $\mathrm{P}_{\mathrm{cov}}^{*}$ ? iii) Is this functional relation an injective function?

Equation (5) is instrumental to answer these questions.

Theorem 1: Let $0 \leq \mathrm{P}_{\text {cov }}^{*} \leq 1$ be the coverage to be fulfilled. It can be achieved if $\mathrm{P}_{\text {cov }}^{*}<\left(1+\Upsilon \mathcal{L}\left(\lambda_{\mathrm{MT}} / \lambda_{\mathrm{BS}}\right)\right)^{-1}$. $\mathrm{P}_{\mathrm{tx}}$ can be formulated as $\mathrm{P}_{\mathrm{tx}}=\kappa \sigma_{\mathrm{N}}^{2} \gamma_{\mathrm{D}} \mathcal{T}\left(\lambda_{\mathrm{BS}}\right)$, where:

$$
\mathcal{T}(\xi)=\left(\frac{-\ln \left(1-\mathrm{P}_{\mathrm{cov}}^{*}\left(1+\Upsilon \mathcal{L}\left(\lambda_{\mathrm{MT}} / \xi\right)\right)\right)}{\pi \xi\left(1+\Upsilon \mathcal{L}\left(\lambda_{\mathrm{MT}} / \xi\right)\right) \Gamma(1+\delta)}\right)^{1 / \delta}
$$

and $\mathcal{T}\left(\xi=\lambda_{\mathrm{BS}}\right)$ is an injective function in $\lambda_{\mathrm{BS}}$.

Proof: Equation (7) follows from (5) by setting $\mathrm{P}_{\text {cov }}\left(\gamma_{\mathrm{D}}, \gamma_{\mathrm{A}}=\gamma_{\mathrm{D}} \Gamma(1+\delta)^{-1 / \delta}\right)=\mathrm{P}_{\text {cov }}^{*}$. The feasibility condition on $\mathrm{P}_{\text {cov }}^{*}$ follows from (7), since the argument of the logarithmic function cannot be negative. The injectivity of $\mathcal{T}(\cdot)$ follows by computing its firstorder derivative and noting that it is non-positive, i.e., $\dot{\mathcal{T}}_{\lambda_{\mathrm{BS}}}\left(\lambda_{\mathrm{BS}}\right) \leq 0$ for $\lambda_{\mathrm{BS}} \geq 0$ and $0 \leq \mathrm{P}_{\text {cov }}^{*}<$ $\left(1+\Upsilon \mathcal{L}\left(\lambda_{\mathrm{MT}} / \lambda_{\mathrm{BS}}\right)\right)^{-1} \leq 1$, since $\dot{\mathcal{L}}_{\lambda_{\mathrm{BS}}}\left(\lambda_{\mathrm{MT}} / \lambda_{\mathrm{BS}}\right) \geq 0$ and $\mathcal{L}\left(\lambda_{\mathrm{MT}} / \lambda_{\mathrm{BS}}\right)+\lambda_{\mathrm{BS}} \dot{\mathcal{L}}_{\lambda_{\mathrm{BS}}}\left(\lambda_{\mathrm{MT}} / \lambda_{\mathrm{BS}}\right) \geq 0$.

Remark 3: For brevity, we focus our attention only on the interplay between $\mathrm{P}_{\mathrm{tx}}$ and $\lambda_{\mathrm{BS}}$, but (5) can be used to study functional relations among any system parameters.

To the best of our knowledge, Theorem 1 constitutes the only available analytical expression that, for cellular networks, provides one with an explicit relation between $\mathrm{P}_{\mathrm{tx}}$ and $\lambda_{\mathrm{BS}}$. To get deeper insight into the relation of $\mathrm{P}_{\mathrm{tx}}$ as a function of $\lambda_{\mathrm{BS}}$, we study, in detail, two relevant asymptotic regimes.

Corollary 1: Let the BSs be sparsely deployed compared with the MTs, i.e., $\lambda_{\mathrm{BS}} / \lambda_{\mathrm{MT}} \ll 1 . \mathcal{T}\left(\xi=\lambda_{\mathrm{BS}}\right)$ is a single variable posynomial function in $\lambda_{\mathrm{BS}}$ of degree $-1 / \delta=-\beta / 2$ :

$$
\mathcal{T}\left(\lambda_{\mathrm{BS}}\right) \approx\left(\frac{-\ln \left(1-\mathrm{P}_{\mathrm{cov}}^{*}(1+\Upsilon)\right)}{\pi(1+\Upsilon) \Gamma(1+\delta)}\right)^{1 / \delta} \lambda_{\mathrm{BS}}^{-1 / \delta}
$$

Proof: If $\lambda_{\mathrm{BS}} / \lambda_{\mathrm{MT}} \ll 1$, then $\mathcal{L}\left(\lambda_{\mathrm{MT}} / \lambda_{\mathrm{BS}}\right) \approx 1$ holds true. The proof follows directly from (7).

Corollary 2: Let the BSs be densely deployed compared with the MTs, i.e., $\lambda_{\mathrm{BS}} / \lambda_{\mathrm{MT}} \gg 1$. Let us assume, in addition, $\left(\lambda_{\mathrm{MT}} / \lambda_{\mathrm{BS}}\right) \Upsilon \ll 1 . \mathcal{T}\left(\xi=\lambda_{\mathrm{BS}}\right)$ is a single variable posynomial function in $\lambda_{\mathrm{BS}}$ of degree $-1 / \delta=-\beta / 2$ :

$$
\mathcal{T}\left(\lambda_{\mathrm{BS}}\right) \approx\left(-\ln \left(1-\mathrm{P}_{\text {cov }}^{*}\right) /(\pi \Gamma(1+\delta))\right)^{1 / \delta} \lambda_{\mathrm{BS}}^{-1 / \delta}
$$


Proof: If $\lambda_{\mathrm{BS}} / \lambda_{\mathrm{MT}} \gg 1$, then $\mathcal{L}\left(\lambda_{\mathrm{MT}} / \lambda_{\mathrm{BS}}\right) \approx \lambda_{\mathrm{MT}} / \lambda_{\mathrm{BS}}$ holds true. If, in addition, $\left(\lambda_{\mathrm{MT}} / \lambda_{\mathrm{BS}}\right) \Upsilon \ll 1$, then $1+$ $\left(\lambda_{\mathrm{MT}} / \lambda_{\mathrm{BS}}\right) \Upsilon \approx 1$. The proof follows from (7).

Remark 4: Corollaries 1 and 2 are in agreement with link budget calculations of isolated wireless links: The transmit power that is necessary to fulfill a given coverage probability requirement is a posynomial increasing function of the average cell radius whose order of growth coincides with the path-loss slope $(\beta)$. This conclusion follows from (8) and (9), by taking into account that the relation between the average cell radius, $\mathrm{R}_{\text {cell }}$, and $\lambda_{\mathrm{BS}}$ is, approximately, $\lambda_{\mathrm{BS}}=1 /\left(\pi \mathrm{R}_{\text {cell }}^{2}\right)$.

\section{PERformance TREndS}

The aim of this section is to study the impact of the most important system parameters, i.e., $\mathrm{P}_{\mathrm{tx}}, \mathrm{B}_{\mathrm{W}}, \lambda_{\mathrm{BS}}, \lambda_{\mathrm{MT}}$, used to optimize cellular networks. The main objective consists of proving that the approximation in (5) allows one to unveil the impact of $\mathrm{P}_{\mathrm{tx}}, \mathrm{B}_{\mathrm{W}}, \lambda_{\mathrm{BS}}, \lambda_{\mathrm{MT}}$ in rigorous mathematical terms. The relevance of this study for cellular network design lies in the difficulty of doing the same, for some system parameters, by direct inspection of (1). Two performance metrics are analyzed: i) the coverage probability in (5) and ii) the Potential Spectral Efficiency (PSE) defined as follows [1, Eq. (9)]:

$$
\widehat{\operatorname{PSE}}\left(\gamma_{\mathrm{D}}\right) \stackrel{(a)}{\approx} \mathrm{B}_{\mathrm{W}} \rho_{\mathrm{D}} \lambda_{\mathrm{BS}} \mathcal{L}\left(\lambda_{\mathrm{MT}} / \lambda_{\mathrm{BS}}\right) \widehat{\mathrm{P}}_{\mathrm{Cov}}\left(\gamma_{\mathrm{D}}\right)
$$

where the approximation in (a) originates from (5), and, for ease of writing, the following notation is used: $\rho_{\mathrm{D}}=\log _{2}\left(1+\gamma_{\mathrm{D}}\right), \widehat{\gamma}_{\mathrm{A}}=\gamma_{\mathrm{D}} \Gamma(1+\delta)^{-1 / \delta}, \widehat{\mathrm{P}}_{\mathrm{cov}}\left(\gamma_{\mathrm{D}}\right)=$ $\mathrm{P}_{\text {cov }}\left(\gamma_{\mathrm{D}}, \widehat{\gamma}_{\mathrm{A}}\right)$, and $\widehat{\operatorname{PSE}}\left(\gamma_{\mathrm{D}}\right)=\operatorname{PSE}\left(\gamma_{\mathrm{D}}, \widehat{\gamma}_{\mathrm{A}}\right)$.

In the sequel, for clarity, the network parameter of interest is denoted by $\zeta$. The other parameters are implicitly assumed to be constant. For ease of notation, accordingly, the shorthand notation $\widehat{\mathrm{P}}_{\text {cov }}\left(\gamma_{\mathrm{D}}\right)=\widehat{\mathrm{P}}_{\mathrm{cov}}\left(\gamma_{\mathrm{D}} ; \zeta\right)=\mathcal{P}(\zeta)$ and $\widehat{\operatorname{PSE}}\left(\gamma_{\mathrm{D}}\right)=$ $\widehat{\mathrm{PSE}}\left(\gamma_{\mathrm{D}} ; \zeta\right)=\mathcal{S}(\zeta)$ for $\mathrm{P}_{\mathrm{cov}}$ and PSE, respectively, is used.

\section{A. Coverage Probability}

The impact of $\mathrm{P}_{\mathrm{tx}}, \mathrm{B}_{\mathrm{W}}, \lambda_{\mathrm{BS}}$, and $\lambda_{\mathrm{MT}}$ on $\mathcal{P}(\cdot)$ is summarized in the following four propositions, respectively.

Proposition 1: Let us consider $\zeta=\mathrm{P}_{\mathrm{tx}}$. The following holds true: i) $\mathcal{P}(\zeta)$ is monotonically increasing in $\zeta$, ii) $\mathcal{P}(\zeta \rightarrow 0)=$ 0 , and iii) $\mathcal{P}(\zeta \rightarrow+\infty)=\left(1+\Upsilon \mathcal{L}\left(\lambda_{\mathrm{MT}} / \lambda_{\mathrm{BS}}\right)\right)^{-1}$.

Proof: It follows by direct inspection of (5).

Remark 5: By direct inspection of (1), the same trend as in Proposition 1 holds true for the SINR-coverage.

Proposition 2: Let us consider $\zeta=\mathrm{B}_{\mathrm{W}}$. The following holds true: i) $\mathcal{P}(\zeta)$ is monotonically decreasing in $\zeta$, ii) $\mathcal{P}(\zeta \rightarrow 0)=\left(1+\Upsilon \mathcal{L}\left(\lambda_{\mathrm{MT}} / \lambda_{\mathrm{BS}}\right)\right)^{-1}$, iii) $\mathcal{P}(\zeta \rightarrow+\infty)=0$.

Proof: It follows by direct inspection of (5).

Remark 6: By direct inspection of (1), the same trend as in Proposition 2 holds true for the SINR-coverage.

Proposition 3: Let us consider $\zeta=\lambda_{\mathrm{BS}}$. The following holds true: i) $\mathcal{P}(\zeta)$ is monotonically increasing in $\zeta$, ii) $\mathcal{P}(\zeta \rightarrow 0)=0$, and iii) $\mathcal{P}(\zeta \rightarrow+\infty)=1$.

Proof: From (5), $\dot{\mathcal{P}}_{\zeta}(\zeta) \geq 0$ holds true if and only if:

$$
\dot{\mathcal{Q}}_{\zeta}(\zeta)(1+\Upsilon \mathcal{L}(\zeta))-\mathcal{Q}(\zeta) \Upsilon \dot{\mathcal{L}}_{\zeta}(\zeta) \geq 0
$$

where $\mathcal{Q}(\zeta)=\mathcal{Q}\left(\zeta, \mathrm{P}_{\mathrm{tx}}, \lambda_{\mathrm{MT}} / \zeta\right)$ and $\mathcal{L}(\zeta)=\mathcal{L}\left(\lambda_{\mathrm{MT}} / \zeta\right)$. (11) is true for $\zeta \geq 0$, since $\mathcal{Q}(\zeta) \geq 0, \dot{\mathcal{Q}}_{\zeta}(\zeta) \geq 0, \mathcal{L}(\zeta) \geq 0$, and $\dot{\mathcal{L}}_{\zeta}(\zeta) \leq 0$ for $\zeta \geq 0$. Then, i) is proved. ii) and iii) follow from $\mathcal{L}(\zeta \rightarrow 0)=1$ and $\mathcal{L}(\zeta \rightarrow+\infty)=0$, respectively.

Remark 7: Due to the lack of a closed-form expression for the SINR-coverage, it is not straightforward to prove, in mathematical terms, the impact of $\lambda_{\mathrm{BS}}$ directly from (1). Since $\dot{\mathcal{L}}\left(\lambda_{\mathrm{MT}} / \zeta\right) \leq 0$, i.e., $\mathcal{L}(\zeta)$ is decreasing in $\zeta$, the same trend as in Proposition 3 holds true for the SIR-coverage.

Proposition 4: Let us consider $\zeta=\lambda_{\mathrm{MT}}$ and denote $\widehat{r}_{\mathrm{A}}=$ $\mathrm{P}_{\mathrm{tx}} /\left(\kappa \sigma_{\mathrm{N}}^{2} \widehat{\gamma}_{\mathrm{A}}\right)$. The following holds true: i) $\mathcal{P}(\zeta)$ is monotonically decreasing in $\zeta$, ii) $\mathcal{P}(\zeta \rightarrow 0)=1-e^{-\pi \lambda_{\mathrm{BS}} \widehat{r}_{\mathrm{A}}^{1 / \delta}}$, and iii) $\mathcal{P}(\zeta \rightarrow+\infty)=\left(1-e^{-\pi \lambda_{\mathrm{BS}} \widehat{r}_{\mathrm{A}}^{1 / \delta}(1+\Upsilon)}\right) /(1+\Upsilon)$.

Proof: From (5), $\dot{\mathcal{P}}_{\zeta}(\zeta) \leq 0$ holds true if and only if:

$$
\Upsilon \dot{\mathcal{L}}_{\zeta}(\zeta)\left[(1+\mathcal{C}(1+\Upsilon \mathcal{L}(\zeta))) e^{-\mathcal{C}(1+\Upsilon \mathcal{L}(\zeta))}-1\right] \leq 0
$$

where $\mathcal{C}=\pi \lambda_{\mathrm{BS}} \widehat{r}_{\mathrm{A}}^{1 / \delta}, \mathcal{Q}(\zeta)=\mathcal{Q}\left(\lambda_{\mathrm{BS}}, \mathrm{P}_{\mathrm{tx}}, \zeta / \lambda_{\mathrm{BS}}\right)$, and $\mathcal{L}(\zeta)=\mathcal{L}\left(\zeta / \lambda_{\mathrm{BS}}\right)$. (12) is true for $\zeta \geq 0$, since $\dot{\mathcal{L}}_{\zeta}(\zeta) \geq 0$ for $\zeta \geq 0$ and the term inside the brackets is negative for $\mathcal{C}(1+\Upsilon \mathcal{L}(\zeta)) \geq 0$. Then, i) is proved. ii) and iii) follow from $\mathcal{L}(\zeta \rightarrow 0)=0$ and $\mathcal{L}(\zeta \rightarrow+\infty)=1$, respectively.

Remark 8: Since $\mathcal{L}\left(\zeta / \lambda_{\mathrm{BS}}\right) \geq 0$ and $\dot{\mathcal{L}}_{\zeta}\left(\zeta / \lambda_{\mathrm{BS}}\right) \geq 0$, i.e., $\mathcal{L}(\zeta)$ is increasing in $\zeta=\lambda_{\mathrm{MT}}$, we evince, from (1), that the SINR-coverage behaves like (5) as a function of $\lambda_{\mathrm{MT}}$.

From Propositions 1-4, a few comments can be made:

- Remarks 5, 6, 8 are readily obtained from (1). They are, however, instrumental to explicitly substantiating that the proposed approximation in (5) yields the same performance trends as (1). The impact of $\lambda_{\mathrm{BS}}$ (see Remark 7) is evaluated in Section IV with Monte Carlo simulations.

- The performance trend as a function of $\lambda_{\mathrm{MT}}$ (see Proposition 4) can be explained as follows: By increasing $\lambda_{\mathrm{MT}}$, the number of active BSs increases, which, in turn, enhances the aggregate other-cell interference, $\mathcal{I}$. As a result, the coverage probability decreases.

- By comparing Proposition 1 against Proposition 3, we evince that network densification, i.e., increasing $\lambda_{\mathrm{BS}}$, has the potential of providing the typical MT with zero outage (the coverage probability tends asymptotically to one as $\left.\lambda_{\mathrm{BS}} \rightarrow+\infty\right)$. This is not possible, on the other hand, by increasing $\mathrm{P}_{\mathrm{tx}}$ : As $\mathrm{P}_{\mathrm{tx}} \rightarrow+\infty$, the coverage probability reaches an upper-limit that is less than one.

\section{B. Potential Spectral Efficiency}

In this section, we turn our attention to the PSE in (10). Unlike the analysis of the coverage in Section III-A, the impact of $\mathrm{B}_{\mathrm{W}}, \lambda_{\mathrm{BS}}, \lambda_{\mathrm{MT}}$ cannot be readily proved for the SINR-PSE, i.e., the PSE obtained by replacing $\widehat{\mathrm{P}}_{\text {cov }}(\cdot)$ in (10) with the SINR-coverage in (1). The reason of the analytical complexity is the presence of the multiplicative factor $\mathrm{B}_{\mathrm{W}} \lambda_{\mathrm{BS}} \mathcal{L}\left(\lambda_{\mathrm{MT}} / \lambda_{\mathrm{BS}}\right)$, which depends on $\mathrm{B}_{\mathrm{W}}, \lambda_{\mathrm{BS}}, \lambda_{\mathrm{MT}}$. Therefore, the performance trends reported in this section are non-trivial, and, to the best of our knowledge, they have not been mathematically and rigorously proved elsewhere.

The impact of $\mathrm{B}_{\mathrm{W}}, \lambda_{\mathrm{BS}}$, and $\lambda_{\mathrm{MT}}$ on $\mathcal{S}(\cdot)$ is summarized in the following three propositions, respectively. The impact of 

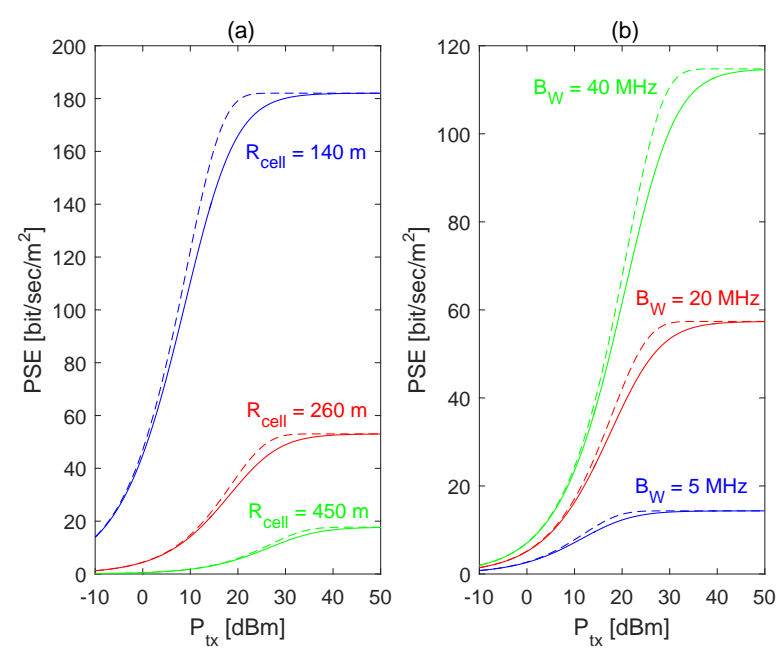

Fig. 1. PSE as a function of $\mathrm{P}_{t x}$ and $\mathrm{R}_{\text {cell }}$ (a) and $\mathrm{B}_{\mathrm{W}}$ (b).

$\mathrm{P}_{\mathrm{tx}}$ is, on the other hand, not discussed in detail. It follows, in fact, by direct inspection of the PSE in (10) and by noting that PSE and coverage have the same behavior. Thus, the trends discussed in Proposition 1 and Remark 5 apply to PSE as well.

Proposition 5: Let us consider $\zeta=\mathrm{B}_{\mathrm{W}}$ and denote $\bar{r}_{\mathrm{A}}=\mathrm{P}_{\mathrm{tx}} /\left(\kappa \mathrm{N}_{0} \widehat{\gamma}_{\mathrm{A}}\right)$. The following holds true: i) $\mathcal{S}(\zeta)$ is monotonically increasing in $\zeta$, ii) $\mathcal{S}(\zeta \rightarrow 0)=0$, and iii) $\mathcal{S}(\zeta \rightarrow+\infty)=+\infty$.

Proof: The PSE is $\mathcal{S}(\zeta)=\mathcal{D} \zeta\left(1-e^{-\mathcal{E} \zeta^{-\delta}}\right)$, where $\mathcal{D}=\rho_{\mathrm{D}} \lambda_{\mathrm{BS}} \mathcal{L}\left(\lambda_{\mathrm{MT}} / \lambda_{\mathrm{BS}}\right) /\left(1+\Upsilon \mathcal{L}\left(\lambda_{\mathrm{MT}} / \lambda_{\mathrm{BS}}\right)\right)$ and $\mathcal{E}=$ $\pi \lambda_{\mathrm{BS}}\left(1+\Upsilon \mathcal{L}\left(\lambda_{\mathrm{MT}} / \lambda_{\mathrm{BS}}\right)\right) \bar{r}_{\mathrm{A}}^{\delta}$. i) follows because $\dot{\mathcal{S}}_{\zeta}(\zeta) \geq$ 0 , since $\dot{\mathcal{S}}_{\zeta}(\zeta \rightarrow 0)=1, \dot{\mathcal{S}}_{\zeta}(\zeta \rightarrow+\infty)=0$, and $\ddot{\mathcal{S}}_{\zeta}(\zeta)=$ $-\delta \mathcal{E D} e^{-\mathcal{E} \zeta^{-\delta}}\left(\delta \mathcal{E} \zeta^{-(1+2 \delta)}+(1-\delta) \zeta^{-(1+\delta)}\right) \leq 0$ for $\beta>2$. ii) directly follows from (10) and iii) is obtained by computing the limit $\mathcal{S}(\zeta \rightarrow+\infty)$ with the aid of De L'Hopital's rule. $\square$

Remark 9: Comparing Propositions 2 and 5, we evince that $\mathrm{P}_{\text {cov }}$ and PSE have opposite trends as a function of $\mathrm{B}_{\mathrm{W}}$.

Proposition 6: Let us consider $\zeta=\lambda_{\mathrm{BS}}$. The following holds true: i) $\mathcal{S}(\zeta)$ is monotonically increasing in $\zeta$, ii) $\mathcal{S}(\zeta \rightarrow 0)=0$, and iii) $\mathcal{S}(\zeta \rightarrow+\infty)=\mathrm{B}_{\mathrm{W}} \rho_{\mathrm{D}} \lambda_{\mathrm{MT}}$.

Proof: i) follows because $\mathcal{P}(\zeta)$ (see Proposition 3) and $\zeta \mathcal{L}\left(\lambda_{\mathrm{MT}} / \zeta\right)$ increase in $\zeta$. ii) and iii) follow from (10) by using Proposition 3 and taking into account that $\zeta \mathcal{L}\left(\lambda_{\mathrm{MT}} / \zeta\right) \rightarrow$ 0 if $\zeta \rightarrow 0$ and $\zeta \mathcal{L}\left(\lambda_{\mathrm{MT}} / \zeta\right) \rightarrow \lambda_{\mathrm{MT}}$ if $\zeta \rightarrow+\infty$.

Proposition 7: Let us consider $\zeta=\lambda_{\mathrm{MT}}$ and denote $\widehat{r}_{\mathrm{A}}=\mathrm{P}_{\mathrm{tx}} /\left(\kappa \sigma_{\mathrm{N}}^{2} \widehat{\gamma}_{\mathrm{A}}\right)$. The following holds true: i) $\mathcal{S}(\zeta)$ is monotonically increasing in $\zeta$, ii) $\mathcal{S}(\zeta \rightarrow 0)=0$, and iii) $\mathcal{S}(\zeta \rightarrow+\infty)=\mathrm{B}_{\mathrm{W}} \rho_{\mathrm{D}} \lambda_{\mathrm{BS}}\left(1-e^{-\pi \lambda_{\mathrm{BS}} \widehat{r}_{\mathrm{A}}^{\delta}(1+\Upsilon)}\right) /(1+\Upsilon)$.

Proof: From (10), $\dot{\mathcal{S}}_{\zeta}(\zeta) \geq 0$ holds true if and only if:

$$
\dot{\mathcal{L}}_{\zeta}(\zeta) \mathcal{Q}(\zeta)+\mathcal{L}(\zeta) \dot{\mathcal{Q}}_{\zeta}(\zeta)+\Upsilon \mathcal{L}^{2}(\zeta) \dot{\mathcal{Q}}_{\zeta}(\zeta) \geq 0
$$

where $\mathcal{Q}(\zeta)=\mathcal{Q}\left(\lambda_{\mathrm{BS}}, \mathrm{P}_{\mathrm{tx}}, \zeta / \lambda_{\mathrm{BS}}\right)$ and $\mathcal{L}(\zeta)=\mathcal{L}\left(\zeta / \lambda_{\mathrm{BS}}\right)$. (13) is true for $\zeta \geq 0$, since $\mathcal{Q}(\zeta) \geq 0, \dot{\mathcal{Q}}_{\zeta}(\zeta) \geq 0, \mathcal{L}(\zeta) \geq 0$, and $\dot{\mathcal{L}}_{\zeta}(\zeta) \geq 0$ for $\zeta \geq 0$. Then, i) is proved. ii) and iii) follow from $\mathcal{L}(\zeta \rightarrow 0)=0$ and $\mathcal{L}(\zeta \rightarrow+\infty)=1$, respectively.

Remark 10: Comparing Propositions 4 and 7, we note that $\mathrm{P}_{\mathrm{cov}}$ and PSE have opposite trends as a function of $\lambda_{\mathrm{MT}}$.
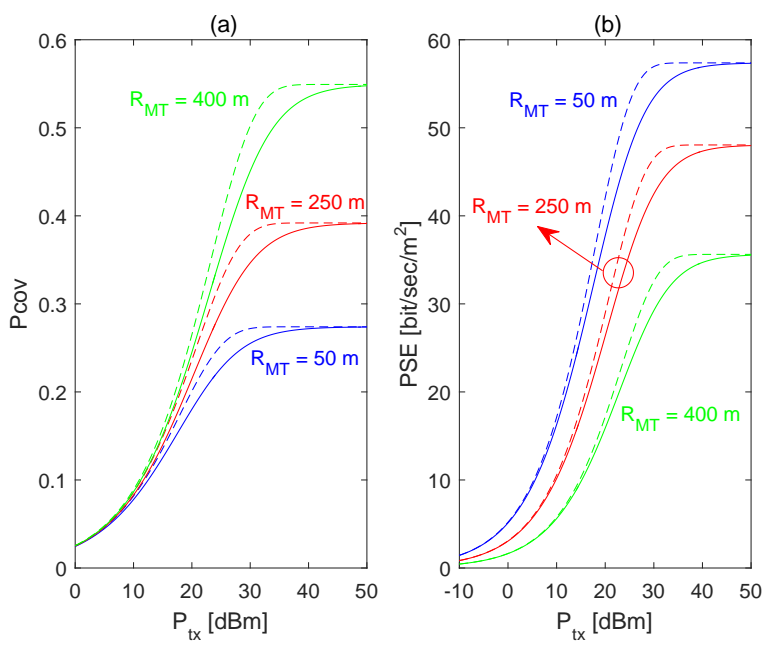

Fig. 2. $\mathrm{P}_{\mathrm{cov}}$ (a) and PSE (b) as a function of $\mathrm{P}_{\mathrm{tx}}$ and $\mathrm{R}_{\mathrm{MT}}$.

\section{NumericAl RESUlts}

The aim of this section it to analyze the accuracy of the approximation in (5) and to substantiate the performance trends proved in Section III as a function of the network parameters $\mathrm{P}_{\mathrm{tx}}, \mathrm{B}_{\mathrm{W}}, \lambda_{\mathrm{BS}}$, and $\lambda_{\mathrm{MT}}$. Unless otherwise stated, the simulation setup is: $\beta=3.5, f_{c}=2.1 \mathrm{GHz}, \kappa=\left(4 \pi f_{c} / 3 \cdot 10^{8}\right)^{2}, \mathrm{~N}_{0}$ $=-174 \mathrm{dBm} / \mathrm{Hz}, \mathrm{B}_{\mathrm{W}}=20 \mathrm{MHz}, \lambda_{\mathrm{BS}}=1 /\left(\pi \mathrm{R}_{\text {cell }}^{2}\right) \mathrm{BSs} / \mathrm{m}^{2}$ with $\mathrm{R}_{\text {cell }}=250 \mathrm{~m}, \lambda_{\mathrm{MT}}=1 /\left(\pi \mathrm{R}_{\mathrm{MT}}^{2}\right)=121 \mathrm{MTs} / \mathrm{km}^{2}$ with $\mathrm{R}_{\mathrm{MT}}=51.29 \mathrm{~m}$, and $\gamma_{\mathrm{D}}=5 \mathrm{~dB}$.

The numerical results are illustrated in Figs. 1 and 2. The solid lines report the SINR-coverage and SINR-PSE obtained from (1) and (10). The dashed lines show the proposed approximation based on (5). The numerical illustrations provide evidence that the proposed approximation is accurate enough for practical applications, and, more importantly, yields the same trends as the widely used SINR-coverage. Therefore, (5) and (10) constitute accurate and tractable analytical frameworks for the analysis and optimization of cellular networks as a function of several network parameters of interest.

\section{CONCLUSION}

In this letter, we have shown that the coverage probability recently introduced in [1] constitutes a closed-form upperbound of the conventional SINR-coverage. The new definition of coverage probability provides one with a tractable analytical framework, from which the impact of the most important system parameters for the design of cellular networks can be rigorously proved. Possible generalizations of this work include the analysis of bounded path-loss models, tri-dimensional network deployments, multi-tier cellular networks.

\section{REFERENCES}

[1] M. Di Renzo, A. Zappone, T. T. Lam, and M. Debbah, "System-level modeling and optimization of the energy efficiency in cellular networks A stochastic geometry framework", IEEE Trans. Wireless Commun., Jan. 2018, to appear. IEEE Early Access (https://arxiv.org/abs/1801.07513).

[2] F. Baccelli and B. Blaszczyszyn, Stochastic Geometry and Wireless Networks, Part I: Theory, Now Publishers, Sep. 2009.

[3] J. G. Andrews, F. Baccelli, and R. K. Ganti, "A tractable approach to coverage and rate in cellular networks", IEEE Trans. Commun., vol. 59 , no. 11, pp. 3122-3134, Nov. 2011. 